\title{
Engenharia Kansei no design automotivo de exterior
}

\section{Kansei engineering in automotive exterior design}

\author{
LIBERATTI, Carolina; Mestranda em Design; PPGDesign Universidade Anhembi Morumbi
}

ca_liberatti@yahoo.com.br

ZUANON, Rachel; Doutora em Design; PPGDesign Universidade Anhembi Morumbi

rachel@zuannon.com.br

\section{Resumo}

O objetivo do presente artigo é realizar uma revisão da literatura do emprego da Engenharia Kansei, enquanto metodologia aplicada ao desenvolvimento de produto, especificamente à área do design automotivo, no período entre 1995 e 2017. O recorte aqui proposto, com enfoque particular no design automotivo de exterior, deve-se ao fator limitante de páginas, sem que, contudo, se perca a profundidade necessária para a compreensão dos 7 estudos de caso apresentados. Almeja-se discorrer sobre a categoria Kansei empregada em cada pesquisa selecionada, junto à análise dos fatores semânticos envolvidos e suas relações com os elementos físicos de design. Além disso, pretende-se preencher a lacuna existente nos âmbitos nacional e internacional quanto à revisão de literatura dedicada ao design automotivo de exterior, de modo a contribuir com subsídios a este campo de investigação no desenvolvimento de pesquisas brasileiras, com a identificação de autores relevantes neste contexto, bem como com o reconhecimento das principais abordagens da referida metodologia centrada no usuário.

Palavras Chave: design automotivo; engenharia Kansei; desenvolvimento de produto.

\begin{abstract}
This paper aims to accomplish a literature revision on regards of automotive design studies in which it was applied Kansei Engineering methodology, on the period between 1995 and 2017. The research focus on exterior automotive design is due to the number of page limitation, without losing deepness needed to comprehend the 7 case studies presented. It aims to demonstrate which Kansei category was used in each selected research and to analyze the involved semantic factors and their relation with physical design elements. Besides that, the research intends to fill the gap in both national and international fields with literature revision dedicated to exterior automotive design, in a way to contribute with Brazilian investigation development by identifying relevant authors and recognizing main approaches of this user center methodology.
\end{abstract}

Keywords: automotive design; Kansei engineering; product development. 


\section{Introdução}

Segundo Bach (2009), o desenvolvimento de mercados globais pautados por avanços tecnológicos em velocidade exponencial, leva a sociedade contemporânea à sobrevivência em um cenário de extremo consumo, no qual a competitividade entre os produtos pela atenção de seus potenciais compradores prepondera.

No mercado automobilístico, a competitividade é fomentada pelas instâncias de customização e personalização, as quais visam agregar diferenciais aos produtos funcionalmente similares. Assim, itens como luxuosidade do material e qualidade do acabamento se sobressaem quando comparados à potência do motor e ao consumo de combustível, como aponta o estudo de Jindo e Hirasago (1997).

Neste sentido, as principais pesquisas voltadas ao design de interior e exterior automotivo indicam o método Kansei (NAGAMACHI, 1995, 2002a, 2002b) como fator estratégico à compreensão das impressões dos usuários sobre determinado produto. Tal metodologia objetiva a tradução das emoções, sentimentos, experiências pessoais e conhecimento de cada indivíduo, na relação com determinado produto, em um modelo matemático capaz de fornecer dados aplicáveis ao contexto de definição dos parâmetros de um projeto, com vistas a soluções de design no referido âmbito.

Schütte $(2002$, p.22) cita a descrição de Nagamachi referente à palavra Kansei como "a impressão subjetiva individual de um certo artefato, ambiente, ou situação, a qual emprega todos os sentidos da visão, audição, olfato, tato e gustação". Como aponta Nagamachi (2002a), o Kansei engloba tanto o sentimento psicológico do consumidor, quanto o seu contexto fisiológico. A palavra Kansei é geralmente traduzida como sensitividade, sensibilidade, sentimento ou necessidade do consumidor em relação a determinado produto. Harada a define como "um processo interno do cérebro envolvido na construção da reação intuitiva ao estímulo externo" como referenciado em Lokman (2010, p.3).

Disso, o método Kansei, além de corroborar às diversas fases de desenvolvimento de um produto, também desempenha papel significativo nos processos de redesign, na avaliação do produto frente aos seus competidores, bem como na antevisão dos potenciais impactos emocionais de um novo conceito/produto a ser lançado no mercado.

Ao associar o campo do design de interior e exterior automotivo ao método Kansei, dezessete pesquisas se destacam: sete com enfoque no design de exterior automotivo NAGAMACHI (1995); SHEN et al. (2000); LAl (2005); CHANG et al. (2006); KIM et al. (2012); YOGASARA \& VALENTINO (2017); SIHOMBING et al. (2017); e dez dedicadas ao design de interior automotivo JINDO \& HIRASAGO (1997); TANOUE et al. (1997); SCHÜTTE \& EKLUND (2005); BAHN et al. (2009); JUNG et al. (2010); KIM et al. (2011); SHEN et al. (2012); GENTNER et al. (2013); MOHAMED \& MUSTAFA (2014) e VIEIRA et al. (2017). Todas elas são desenvolvidas em território internacional (Bélgica, Coréia do Sul, Estados Unidos, França, Indonésia, Japão, Malásia, Portugal, Reino Unido e Taiwan), no período compreendido entre 1995 e 2017.

Diante deste cenário e da ausência de uma revisão da literatura dedicada ao assunto, seja em idioma estrangeiro ou português, o presente artigo se propõe a contribuir com a redução desta lacuna e com subsídios ao desenvolvimento de pesquisas nacionais relacionadas a este assunto, ao sistematizar e articular as referências supracitadas. 


\section{Tipos de Kansei Engineering}

A estrutura e o método Kansei são consistentes, mas suas ferramentas sofrem modificações ao longo dos anos, uma vez que transitam por áreas distintas, tais como matemática, estatística, engenharia, marketing, ciência da computação e psicologia. Segundo estudos (Lokman, 2010) existem 8 tipos diferentes de Engenharia Kansei (EK):

Quadro 1 - Tipos de KE

\begin{tabular}{lll}
\hline Tipos de KE & Classification & Tradução \\
\hline Tipo I & Category Classification & Classificação de Categorias \\
Tipo II & Kansei Engineering Computer System (KES) & EK Sistemas de Computador \\
Tipo III & Kansei Engineering Modeling & EK Modelos Matemáticos \\
Tipo IV & Hybrid Kansei Engineering & EK Híbrido \\
Tipo V & Virtual Kansei Engineering (VIKE) & EK Virtual \\
Tipo VI & Collaborative Kansei Engineering & EK Colaborativo \\
Tipo VII & Concurrent Kansei Engineering & EK Simultâneo \\
Tipo VIII & Rough Sets Kansei Engineering & EK Conjuntos Aproximados \\
\hline
\end{tabular}

Fonte: adaptado de Lokman (2010)

Tipo I - Classificação de Categorias (Category Classification) é caracterizado por uma estrutura hierárquica de árvore, e que envolve pesquisa qualitativa. Parte-se de um conceito central, e realiza-se o desdobramento em subconceitos mais específicos. Estes são, então, testados quanto aos aspectos ergonômicos e, posteriormente, detalhados em especificações técnicas do produto. A pesquisa do estilo de vida e comportamento do público-alvo gera palavras conceito, que são reduzidas por diferencial semântico a palavras Kansei, exploradas em subconceitos até que se alcance a correlação com os elementos e parâmetros de design. Neste contexto, é possível citar o desenvolvimento do carro esportivo Mazda Miata (Nagamachi, 1995; 2002a,b), cuja palavra Kansei "integração homem-máquina" foi relacionada ao comprimento do veículo e à ausência de bancos traseiros, como demonstrado mais adiante.

Tipo II - EK Sistemas de Computador (Kansei Engineering Computer System - KES) consiste em um sistema assistido por computador, que utiliza estatísticas matemáticas para definir regras relacionais entre palavras Kansei e imagens do produto. As palavras Kansei inseridas no sistema são correlacionadas ao banco de imagens, e programadas para indicar o design mais adequado ao referente conceito. Como aponta Nagamachi (2002b), esse sistema proporciona ao consumidor a escolha do produto mais adequado a sua necessidade, ao passo que conscientiza o time de desenvolvimento do referido produto sobre a sugestão do sistema. Um exemplo de KES é o software 
HULIS, aprovado pelo Ministério da Construção do Japão, o qual seleciona o projeto de cozinha e design baseados no estilo de vida e hábitos do cliente, como apresentado no estudo de Imamura citado em Gabardo (2014).

Tipo III - EK Modelos Matemáticos (Kansei Engineering Modeling) refere-se à construção de modelos matemáticos para serem aplicados em modelos preditivos de engenharia Kansei. Por exemplo, Fukushima et al. (1995) em um estudo para a marca Sanyo, incorporaram a percepção sensorial humana ao método de processamento de imagens, para obter uma cópia a cores e impressa visualmente mais atraente se comparada à imagem original. A pesquisa explorou a viabilidade de tradução quantitativa da percepção sensorial humana, com a utilização do controle de cor seletiva a partir do modelo matemático da escala de cor RGB, a fim de reproduzir-se o tom de pele japonês mais desejável.

Tipo IV - EK Híbrido ou Engenharia Kansei Reversa (Hybrid Kansei Engineering), opera em um conjunto de dois sistemas: o de engenharia Kansei (KES), também denominado forward; e o reverso, ou backward. O objetivo é auxiliar o designer na tomada de decisões sobre o produto. Essa metodologia apoia-se em um sistema de base de dados informatizado, no qual é possível inserir palavras Kansei para se obter sugestões de produtos (KES), ou inserir desenhos de produtos com o objetivo de que expressões Kansei sejam atribuídas a eles (Kansei reverso). Esse sistema apresenta algumas vantagens, tais como: uma rápida estimativa da opinião do consumidor sobre o conceito; não requer a exposição de conceitos e protótipos para potenciais usuários; e não demanda elevado nível de recurso de marketing para a realização da pesquisa. Como exemplo, é possível citar a pesquisa de Jindo e Hirasago (1997) referente à aplicação do sistema de engenharia Kansei reversa para o desenvolvimento do volante de carro de passeio da marca Nissan. Quando se digita um adjetivo no sistema informatizado, filtram-se as imagens do banco de dados referente ao relativo conceito, apresentando-as em ordem decrescente de prioridade.

Tipo V - EK Virtual (Virtual Kansei Engineering - VIKE) configura-se na combinação do sistema de engenharia Kansei Tipo II (KES) - no qual há a base de dados informatizada que traduz as palavras Kansei do consumidor em elementos de design -, com um segundo sistema que conta com o suporte da realidade virtual. Tal combinação proporciona ao usuário uma experiência espacial virtual, com manipulação direta do produto. Como sugere Nagamachi (2002a), o encontro entre a tecnologia de realidade virtual e a engenharia Kansei pode trazer contribuições significativas quando aplicado ao design de ambientes, interior automotivo, design urbano e construção civil. Como exemplo, é possível citar o sistema computadorizado "HousMall" empregado no desenvolvimento de casas japonesas. Este viabiliza a comunicação do usuário, por meio da correlação entre as palavras Kansei - provenientes de pesquisa de opinião com consumidores - e a análise do banco de imagens, a ser avaliada por designers de interior (Nagamachi, 2002a).

Tipo VI - EK Colaborativo (Collaborative Kansei Engineering) conta com um sistema de base de dados acessível pela internet, o qual fomenta o trabalho colaborativo entre designers de diversas localidades, que atuam em um mesmo projeto. Este sistema pode ser utilizado no desenvolvimento e correção de propostas de design, como também auxiliar a comunicação entre designers e clientes por meio da interação remota. Como exemplo, destaca-se o estudo do Sistema de Design Colaborativo pela Internet de Ishihara et al. (2005) citado por Lokman (2010).

Tipo VII - EK Simultâneo (Concurrent Kansei Engineering) caracteriza-se pela participação simultânea de cada área da empresa desenvolvedora do produto, desde o início do projeto. Como 
aponta Nagamachi (2002a), as vantagens da engenharia concurrent são que todas as áreas envolvidas estarão alinhadas quanto à execução, avaliação e análise das palavras Kansei do projeto e, a partir disso, poderão atuar de modo ativo e colaborativo. Essa abordagem viabiliza uma perspectiva holística do design de produto, tanto dos aspectos que envolvem a engenharia e a qualidade deste, quanto aqueles relacionados ao marketing. Destaca-se aqui a pesquisa dirigida ao design de embalagem para cosmético da marca Milbon (Nagamachi, 2002a). Nesse estudo - focado no desenvolvimento de embalagens de xampu e tratamento capilar para mulheres - as áreas de marketing, planejamento do produto, pesquisa, design, produção, vendas e ergonomia foram engajadas no projeto desde o seu início. Como resultado, o design e o material das novas embalagens traduziram o conceito nível zero "maciez e brilho".

Tipo VIII - EK Conjuntos Aproximados (Rough Sets Kansei Engineering) fundado pelo Dr. Z. Pawlak, que lida com dados ambíguos, incertos, lineares e não lineares e, diferentemente do método estatístico, o Rough Sets pode encontrar regras com significado em grupos ao seguir o pensamento: se... então, como aponta Nagamachi (2007). Exemplo da aplicação desse método pode ser encontrado no estudo de Okamoto (2007) sobre o design de lata de cerveja, no qual os elementos de projeto, tais como: marca; rótulo; cor; textura; dentre outros, são analisados simultaneamente e correlacionados às palavras Kansei leveza, design único e atraente.

\section{Aplicações do estudo Kansei na indústria automotiva}

O presente artigo parte do levantamento de artigos científicos disponíveis em bases de dados online, os quais dispõem os termos "engenharia Kansei" ou "engenharia afetiva", associados à designação "design automotivo" ou derivadas (veículo, carro, automóvel), seja em seu título ou em suas palavras-chave.

No período compreendido entre os anos de 1995 e de 2017, são localizados 17 artigos referentes ao desenvolvimento do design automotivo com a aplicação da metodologia Kansei. Destes, 7 referem-se ao design de exterior e os demais relacionam-se ao design de interior. Como já mencionado, enfoca-se aqui o design automotivo de exterior, em razão do limite de páginas indicado por este congresso. Todavia, sem poupar esforços, tampouco densidade e profundidade a sua abordagem. Importa ainda ressaltar que o escopo tratado contempla apenas o emprego da Engenharia Kansei Tipo I - Classificação de Categorias. A este fato corrobora a afirmação de Schütte et al. (2004), quando discorre sobre a pluralidade de ferramentas que constituem a metodologia Kansei, as quais são provenientes de diversas áreas do conhecimento científico e tecnológico. Disso advém a dificuldade de aplicação dos demais tipos de EK ao design automotivo de exterior, principalmente em razão destes empregarem ferramentas que demandam consideráveis investimentos de tempo e de recursos, o que afeta sobremaneira a duração e a validade dos estudos envolvidos.

Pode-se observar a diversidade de aplicações da EK Tipo I por seu emprego nos mais variados contextos, tais como: o estudo de Nagamachi et al. (1995), que aplicou a metodologia na análise da configuração de um carro esportivo para atender as necessidades dos consumidores jovens japoneses; as pesquisas lideradas por Shen et al. (2000), as quais enfocam os elementos de design externo e frontal que mais influenciam a percepção humana; a investigação semântica de Chang et al. (2006), centralizada nas expressões habitualmente usadas pelo consumidor para descrever a forma da silhueta do carro idealizado, enquanto técnica de investigação para melhorar a 
comunicação entre designers e consumidores, e de modo a entregar subsídios à compreensão dessas expressões; as análises de Kim et al. (2012) referentes a possíveis divergências na avaliação dos consumidores quanto ao design externo do automóvel, quando em contato com uma vista do objeto (frontal) ou duas vistas do mesmo objeto (frontal e lateral); a investigação de Yogasara e Valentino (2017) que analisa o mercado automotivo da Indonésia para propor um modelo hatchback alinhado às necessidades do consumidor; e a pesquisa de Sihombing et al. (2017) circunscrita no mercado da Malásia e dedicada à classificação das palavras Kansei para os segmentos automotivos sedan, MPV e city, com o objetivo de evidenciar discrepâncias entre estas palavras e as impressões do usuário, dependendo das vistas frontal, lateral e traseira apresentadas. Em contrapartida, apenas a pesquisa de Lai et al. (2005) retrata o método Taguchi de análise estatística de variação no redesign da silhueta externa do carro. Tal metodologia visa reduzir a discrepância entre o sentimento do consumidor e a concepção intencionada ao projeto, bem como minimizar a disparidade nas respostas emocionais advindas desses consumidores, quando em contato com o estímulo de um mesmo objeto.

Abaixo segue um quadro síntese dos estudos previamente descritos que relacionam palavras Kansei e design automotivo de exterior, compreendidos entre 1995 e 2017. Dada a multiplicidade do método, observa-se que a metodologia Kansei Tipo I foi aplicada com finalidades distintas nos sete artigos selecionados. Isso advém do fato deste articular diversas áreas do conhecimento, e ao mesmo tempo apresentar menor complexidade quando comparado ao emprego dos outros tipos de engenharia Kansei.

Quadro 2 - Artigos publicados no período 1995-2017, relacionados a design de exterior.

\begin{tabular}{|c|c|c|c|}
\hline Pesquisador & Objeto de estudo & Palavras kansei & Elementos de Design \\
\hline $\begin{array}{l}\text { NAGAMACHI } \\
(1995)\end{array}$ & $\begin{array}{l}\text { Analisar a configuração ideal } \\
\text { de um carro esportivo de } \\
\text { baixo custo para o público } \\
\text { masculino jovem }\end{array}$ & $\begin{array}{l}\text { Motorista integrado ao } \\
\text { carro }\end{array}$ & $\begin{array}{l}\text { Comprimento do carro } \\
\text { e configuração interna } \\
\text { com dois bancos }\end{array}$ \\
\hline $\begin{array}{l}\text { SHEN et al. } \\
(2000)\end{array}$ & $\begin{array}{l}\text { Identificar quais elementos do } \\
\text { design frontal influenciam a } \\
\text { percepção humana; e se há } \\
\text { divergência na percepção de } \\
\text { consumidores de } 3 \text { países } \\
\text { distintos (EUA, UK e Japão). }\end{array}$ & $\begin{array}{l}\text { Valor (luxo), } \\
\text { performance } \\
\text { (esportivo), tradição } \\
\text { (clássico e simples), } \\
\text { agradável, arrojado, } \\
\text { aerodinâmica e } \\
\text { iluminação }\end{array}$ & $\begin{array}{l}\text { Vista frontal, farol, } \\
\text { grade dianteira }\end{array}$ \\
\hline $\begin{array}{l}\text { LAI } \\
(2005)\end{array}$ & $\begin{array}{l}\text { Redesign da silhueta do } \\
\text { exterior de carro de } \\
\text { passageiro, com vistas a } \\
\text { reduzir a discrepância e a } \\
\text { ambiguidade da qualidade } \\
\text { percebida do produto, assim } \\
\text { como minimizar a influência } \\
\text { de características individuais }\end{array}$ & $\begin{array}{l}\text { Jovem, atividade ao ar } \\
\text { livre e família }\end{array}$ & $\begin{array}{l}\text { Combinações das } \\
\text { variações de: } \\
\text { entre-eixos, ângulo de } \\
\text { ataque, altura do carro }\end{array}$ \\
\hline $\begin{array}{l}\text { CHANG et al. } \\
(2006)\end{array}$ & $\begin{array}{l}\text { Compreender o desejo oculto } \\
\text { relacionado à forma lateral do } \\
\text { carro, por meio do estudo de }\end{array}$ & $\begin{array}{l}5 \text { meios para } \\
\text { descrever a forma: }\end{array}$ & Não se aplica \\
\hline
\end{tabular}




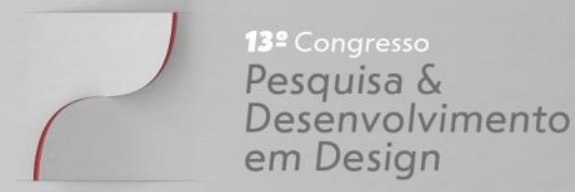

Artigo Completo

\begin{tabular}{|c|c|c|c|}
\hline & $\begin{array}{l}\text { expressões usadas pelo } \\
\text { consumidor para descrever ao } \\
\text { designer o carro imaginado }\end{array}$ & $\begin{array}{l}\text { explicação da forma, } \\
\text { analogia, classificação, } \\
\text { transmissão de } \\
\text { emoções e associação }\end{array}$ & \\
\hline $\begin{array}{l}\text { KIM et al. } \\
(2012)\end{array}$ & $\begin{array}{l}\text { Respectivamente por meio } \\
\text { das estratégias Uninominal } \\
\text { Reality Sets e Binominal } \\
\text { Reality Sets, investigar se a } \\
\text { avaliação do produto pelos } \\
\text { usuários ocorre da mesma } \\
\text { forma para uma imagem do } \\
\text { produto (lateral do carro) e } \\
\text { outras duas imagens do } \\
\text { mesmo produto (duas vistas } \\
\text { do carro). }\end{array}$ & $\begin{array}{l}\text { Intuição: preferência e } \\
\text { estética } \\
\text { Decisão: decisão de } \\
\text { compra, familiaridade } \\
\text { e conhecimento } \\
\text { Emoção: prazer e } \\
\text { entusiasmo }\end{array}$ & $\begin{array}{l}\text { Vista dianteira e } \\
\text { lateral, bem como } \\
\text { combinação de } \\
\text { elementos por } \\
\text { preferência }\end{array}$ \\
\hline $\begin{array}{l}\text { YOGASARA; } \\
\text { VALENTINO } \\
(2017)\end{array}$ & $\begin{array}{l}\text { Proposta de design de exterior } \\
\text { para hatchback, direcionada } \\
\text { ao mercado da Indonésia e } \\
\text { que considera os aspectos } \\
\text { emocionais do consumidor }\end{array}$ & $\begin{array}{l}\text { luxuoso, esportivo, } \\
\text { estético, inovador, } \\
\text { flexível, complexo, } \\
\text { confiável, masculino, } \\
\text { satisfatório }\end{array}$ & $\begin{array}{l}\text { Lanterna, porta-malas, } \\
\text { roda, párachoques } \\
\text { dianteiro e traseiro, } \\
\text { grade, farol, capô, } \\
\text { párabrisa, teto e portas }\end{array}$ \\
\hline $\begin{array}{l}\text { SIHOMBING et } \\
\text { al. } \\
(2017)\end{array}$ & $\begin{array}{l}\text { Investigar a relação das } \\
\text { palavras Kansei com os } \\
\text { segmentos MPV, city e sedan; } \\
\text { e associadas às seguintes } \\
\text { vistas: frontal, lateral e } \\
\text { traseira }\end{array}$ & $\begin{array}{l}\text { estilo, } \\
\text { conforto, } \\
\text { segurança, } \\
\text { luxo e } \\
\text { esportividade }\end{array}$ & $\begin{array}{l}\text { Vista lateral do MPV e } \\
\text { city } \\
\text { Vista lateral do sedan }\end{array}$ \\
\hline
\end{tabular}

Fonte: as autoras (2018)

\section{Estudos de caso}

Abaixo segue pormenorizado os sete artigos que exploraram a metodologia Kansei Engineering, aplicando-a ao design automotivo de exterior.

Nesse contexto, o projeto pioneiro e mais expressivo foi o desenvolvimento do carro esportivo Mazda Miata, pelo pesquisador Nagamachi (1995; 2002a,b), baseado na EK Tipo I Classificação por Categoria com o conceito nível-zero Human Machine Unity (HMU), o qual pode ser traduzido como integração motorista - máquina. Tal conceito foi formulado a partir de pesquisa realizada por meio da aplicação de questionário direcionado a obter informações relacionadas ao estilo de vida e comportamento de jovens motoristas. Todos os dados foram analisados e resultaram em um total de 600 palavras Kansei. Estas foram posteriormente distribuídas em 20 grupos, e organizadas hierarquicamente em níveis de conceito, tais como: enclausuramento, velocidade e comunicação. Cada um destes conceitos também foi desmembrado em subconceitos. Como resultado aos parâmetros de Design, foi possível correlacionar o HMU ao comprimento do carro (próximo a 4 metros) e a configuração interna de dois bancos (motorista e passageiro).

Quadro 3 - Desdobramentos do conceito nível zero do KE para a concepção do modelo Mazda Miata. 


\begin{tabular}{|c|c|c|c|c|c|c|}
\hline Zero & 10 & 20 & 30 & Aspectos físicos & Ergonomia & Engenharia \\
\hline \multirow{4}{*}{$\mathrm{HMU}$} & Enclausuramento & $\begin{array}{l}\cdots \\
\ldots\end{array}$ & & $\begin{array}{l}\text { Tamanho } \\
\text { Altura } \\
\text { Largura }\end{array}$ & $\begin{array}{l}\text { Experimento de } \\
\text { enclausuramento }\end{array}$ & $\begin{array}{l}\text { Design do } \\
\text { chassi, design } \\
\text { do interior }\end{array}$ \\
\hline & Sentimento imediato & $\begin{array}{l}\cdots \\
\ldots\end{array}$ & & $\begin{array}{l}\text { Banco } \\
\text { Volante }\end{array}$ & $\begin{array}{l}\text { Funções do } \\
\text { volante }\end{array}$ & $\begin{array}{l}\text { Power train, } \\
\text { Raio do } \\
\text { volante }\end{array}$ \\
\hline & Velocidade & $\begin{array}{l}\cdots \\
\ldots\end{array}$ & & $\begin{array}{l}\text { Câmbio } \\
\text { Velocímetro } \\
\text { Frequência }\end{array}$ & $\begin{array}{l}\text { Gravidade } \\
\text { negativa }\end{array}$ & $\begin{array}{l}\text { Design do } \\
\text { câmbio e do } \\
\text { velocímetro }\end{array}$ \\
\hline & Comunicação & ... & & Estilo aberto & $\begin{array}{l}\text { Frequência de } \\
\text { ruído }\end{array}$ & Escapamento \\
\hline
\end{tabular}

Fonte: Nagamachi (2002a)

Segundo Nagamachi (2002a), outras montadoras como Nissan, Mitsubishi, Toyota, Honda, Ford, Hyundai e Delphi Systems, além da Mazda, utilizaram a metodologia de engenharia Kansei para o desenvolvimento de carros de passageiros.

Shen et al. (2000) em conjunto à Sprint PCS, dos Estados Unidos, à Universidade Kagawa e ao Instituto Kure, ambos do Japão, e à Universidade Nottingham, do Reino Unido, realizaram um estudo referente à máscara frontal do carro e conduzido com 20 participantes. Deste total, foram selecionados 10 homens e 10 mulheres de cada um dos três países mencionados. Cada participante analisou um total de 24 fotos coloridas da vista frontal do carro de passageiro de 7 marcas distintas, em uma escala de 5 pontos. A pesquisa empregou a metodologia de engenharia Kansei, de modo a relacionar as palavras "valor" (luxuoso, preço, consumo de combustível, potência do motor), "performance" (esportivo, aerodinâmica, beleza) e "tradição" (clássico e simples) aos elementos de design frontais, tais como: forma e padrão da grade; forma e tipo do farol; emblema e forma geral. O objetivo do estudo concentrou-se na identificação dos elementos de design da máscara frontal, e como estes influenciam a percepção humana, com o objetivo de identificar possíveis divergências entre a percepção desses participantes, em vista das diferenças geográficas e culturais de origem. Por fim, identificou-se que o farol e a grade são os elementos que mais influenciam o design frontal, e concluiu-se que não há diferença significativa entre os resultados obtidos junto aos três países, quanto às palavras Kansei relacionadas à percepção frontal do veículo.

Quadro 4 - Valores das palavras Kansei "luxo", "esportivo" e "simples", suas correlações com elementos de design da máscara frontal do automóvel e a avaliação nos três países distintos.

\begin{tabular}{lllll}
\hline $\begin{array}{l}\text { Palavra } \\
\text { Kansei }\end{array}$ & Elemento & EUA & Japão & UK \\
\hline Luxo & Forma: Integral 1 & 0.784 & 0.740 & 0.762 \\
& Tipo: Grade frontal & 0.666 & 0.661 & 0.867 \\
\hline
\end{tabular}




\begin{tabular}{lllll}
\hline & Forma: Integral 2 & 0.657 & 0.671 & 0.790 \\
\hline \multirow{2}{*}{ Esportivo } & Forma: Integral 1 & 0.858 & 0.892 & 0.856 \\
& Forma: Farol & 0.869 & 0.738 & 0.891 \\
& Forma: Grade frontal & 0.763 & 0.754 & 0.811 \\
\hline \multirow{2}{*}{ Simples } & Forma: Integral 1 & & 0.674 & 0.778 \\
& Forma: Farol & & 0.664 & \\
& Forma: Grade frontal & & 0.624 & 0.624 \\
& Indicador de direção & 0.473 & & 0.782 \\
& Emblema & 0.431 & & \\
& Tipo: Farol & 0.428 & & \\
& & &
\end{tabular}

Fonte: adaptado de Shen et al. (2000)

Lai et al. (2005) e Chang et al. (2006), ambos pesquisadores da Universidade Cheng Kung (Taiwan), aplicaram respectivamente o método estatístico Taguchi e a engenharia Kansei na investigação da silhueta do exterior de um carro. Em seus estudos, Lai et al. (2005) objetivaram o aumento da qualidade da experiência do potencial usuário com o produto pretendido em duas instâncias: ao reduzir a discrepância entre o sentimento expresso pelo consumidor e a concepção intencionada pelo projeto; e ao minimizar a variação dos sentimentos entre os diversos consumidores, quando em contato com os estímulos advindos do mesmo objeto. Para tanto, a obtenção de parâmetros de design foi direcionada a evocar as palavras "jovem", "atividade ao ar livre" e "família". Para isso, 6 especialistas, empregaram uma escala de 9 pontos, com vistas à análise de 3 designs e 27 combinações impressas, que contavam com variações de distância entre-eixos, ângulo de ataque e altura em relação ao solo. Como resultado, quando comparado ao design original $(11,12,13)$, as propostas de redesign evocaram um aumento de qualidade por reduzirem em: $41,31 \%$ a discrepância entre o sentimento expresso pelo consumidor e a expectativa intencionada pelo projeto e $51,49 \%$ a ambiguidade, ou seja, as variações de sentimento frente ao mesmo objeto. De acordo com os autores, este estudo pode ser aplicado para redesenhar produtos e adequá-los aos sentimentos de qualidade pré-estabelecidos; modificar produtos com ampliação do atual público-alvo (inserção de mulher jovem ao escopo até então formado por homem maduro); desenhar novos modelos a serem inseridos em uma família de produtos já existente (nova geração de carro, mas que preserve a identidade da família).

Figura 1 - Redesign da proposta inicial de acordo com o estudo Kansei.

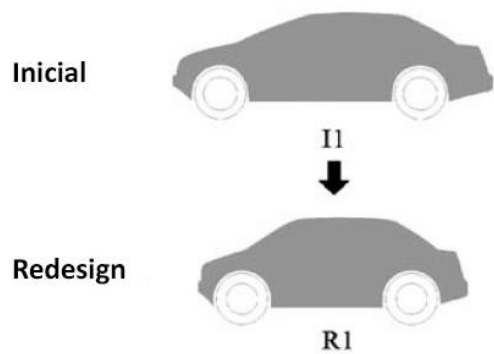

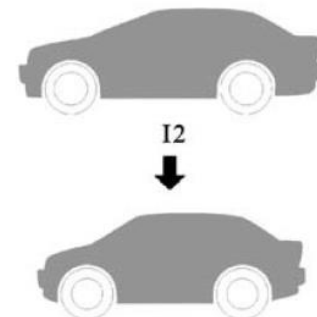

R2

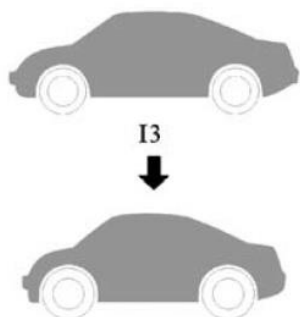

R3

Fonte: adaptado de Lai et al. (2005) 
Já a investigação conduzida por Chang et al. (2006) visa a compreensão das expressões orais usadas por 23 estudantes, sendo 12 homens e 11 mulheres, cuja área de aprendizado não se relacionava ao campo do Design. Para descrever, ao time de desenvolvimento de produto, a silhueta de um carro imaginado e desejado por estes estudantes, foram empregadas em média 30-45 expressões, agrupadas em 5 grupos denominados: explicação da forma; classificação; analogia; transmissão de emoção; e associação. Observa-se que nas fases iniciais do desenvolvimento, são utilizadas expressões de cunho mais abstrato, por relações de analogia, tais como: objetos de forma similar; analogia de forma e cores; associação com lugares; características do usuário; e função. Já nas fases posteriores, são empregados elementos de descrição mais objetivos e tangíveis quanto à forma do produto, tais como indicações de proporção e contorno. Esse estudo visa contribuir ao entendimento do time de desenvolvimento sobre as melhores possibilidades para a forma do produto, por meio de expressões exteriorizadas pelo consumidor.

Tabela 1 - Número de descrições em cada fase do desenvolvimento.

\begin{tabular}{lccccccccccc}
\hline & \multicolumn{7}{c}{ Fase do desenvolvimento } & & \\
\cline { 2 - 10 } & $\mathbf{1}$ & $\mathbf{2}$ & $\mathbf{3}$ & $\mathbf{4}$ & $\mathbf{5}$ & $\mathbf{6}$ & $\mathbf{7}$ & $\mathbf{8}$ & $\mathbf{9}$ & $\mathbf{1 0}$ & Total \\
\hline Explicando a forma & 8 & 13 & 15 & 15 & 20 & 31 & 36 & 41 & 46 & 52 & 257 \\
\hline Classificando & 17 & 18 & 14 & 15 & 18 & 15 & 20 & 15 & 14 & 13 & 159 \\
\hline Analogia & 16 & 17 & 15 & 17 & 20 & 19 & 16 & 15 & 13 & 8 & 156 \\
\hline $\begin{array}{l}\text { Transmissão de } \\
\text { emoção }\end{array}$ & 20 & 16 & 21 & 20 & 17 & 9 & 5 & 5 & 4 & 5 & 122 \\
\hline Associação & 20 & 17 & 17 & 14 & 7 & 7 & 5 & 5 & 2 & 0 & 94 \\
\hline Outros & 1 & 0 & 0 & 0 & 0 & 0 & 0 & 0 & 2 & 4 & 7 \\
\hline Total & 82 & 81 & 82 & 81 & 82 & 81 & 82 & 81 & 81 & 82 & 815 \\
\hline
\end{tabular}

Fonte: adaptado de Chang (2006)

Kim, S. et al. (2012), em colaboração com as universidades de Hiroshima e Tsukuba, partiram de um estudo com 20 estudantes (11 homens e 9 mulheres) de outras áreas não correlatas ao Design, para investigar a relação entre a preferência e a avaliação de produto por meio de imagem. Em um primeiro momento, os participantes indicaram sua preferência ao combinar os elementos de 6 carros de segmentos variados (coupé, sedan e hatchback). Posteriormente, as 36 combinações com imagens do carro em vista única (frontal), e 36 montagens com imagens das duas vistas (lateral e frontal) do mesmo carro foram submetidas à avaliação destes estudantes, a partir das palavras Kansei "intuição" (preferência e estética), "decisão" (decisão de compra, familiaridade e conhecimento) e "emoção" (prazer e entusiasmo). O estudo confirmou que a forma de expor as imagens do objeto afeta a preferência durante a avaliação do produto, uma vez que os elementos rearranjados (ex. farol) influenciam a preferência na imagem frontal, mas não acarretam mudanças na avaliação por duas imagens (frontal e lateral). Essa constatação pode contribuir para elucidar mudanças de opinião do participante durante o julgamento de um produto. 
Figura 2 - Resultado obtido com imagem frontal e com imagem frontal e lateral, respectivamente.
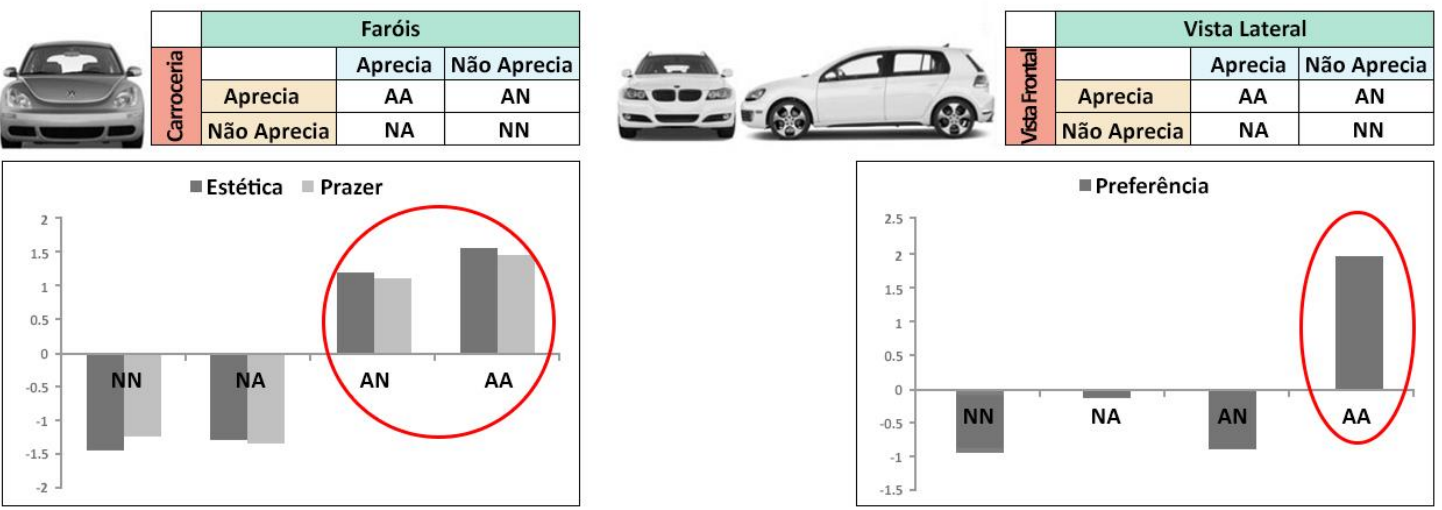

Fonte: adaptado de Kim et al. (2012)

A investigação de Yogasara e Valentino (2017) da Universidade de Parahyangan, na Indonésia, focou o entendimento dos principais requisitos para o desenvolvimento de um carro de passeio para o mercado nacional da Indonésia, empregando-se a metodologia EK Tipo I. A pesquisa bibliográfica associada às entrevistas com os participantes resultaram no levantamento de 164 palavras Kansei, categorizadas em nove grupos: "luxuosidade"; "esportividade"; "estética"; "inovação"; "flexibilidade"; "complexidade"; "confiança"; "masculinidade"; "satisfação", e analisadas em uma escala de 5 pontos. Quarenta e seis homens e dezessete mulheres analisaram, por meio de questionário online, a combinação dos componentes (farol, para-choque, portas e porta-malas) de 16 carros com as palavras Kansei, em um processo colaborativo com o designer de produto. Tal ação resultou no design de um hatchback de 4 portas, posteriormente avaliado por 18 homens e 15 mulheres, que atendeu aos requisitos emocionais dos potenciais usuários.

Tabela 2 - Matriz com a relação entre os elementos de exterior e as palavras Kansei.

\begin{tabular}{|c|c|c|c|c|c|c|c|c|c|c|}
\hline \multirow[t]{2}{*}{ № } & \multirow{2}{*}{$\begin{array}{c}\text { Componentes de } \\
\text { Exterior }\end{array}$} & \multicolumn{9}{|c|}{ Grupos de palavras Kansei } \\
\hline & & 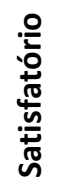 & 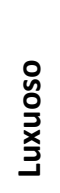 & 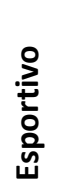 & 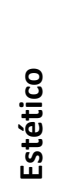 & 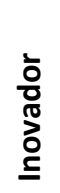 & 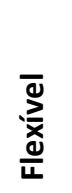 & $\frac{\text { o }}{\frac{x}{0}}$ & 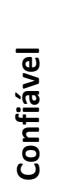 & 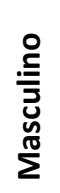 \\
\hline 1 & Lanterna & $x$ & & $x$ & $x$ & $x$ & & & & \\
\hline 2 & Porta-malas & $\mathrm{x}$ & & & & & & & $x$ & \\
\hline 3 & Luz Traseira & $x$ & & $x$ & $x$ & & & & & \\
\hline 4 & Diâmetro do Pneu & $x$ & & $x$ & & & $x$ & & & \\
\hline 5 & Para-choque Traseiro & $X$ & & $x$ & $\mathrm{X}$ & & $\mathrm{x}$ & & $\mathrm{X}$ & \\
\hline 6 & Ângulo de Ataque & $x$ & & & & & $x$ & & $x$ & \\
\hline 7 & Para-choque Dianteiro & & & $x$ & $x$ & & & $x$ & & $\mathrm{X}$ \\
\hline
\end{tabular}




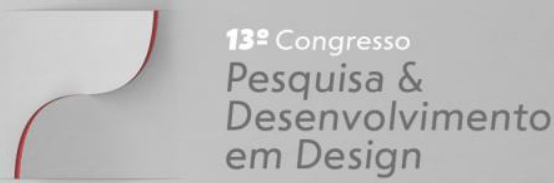

Artigo Completo

\begin{tabular}{|c|c|c|c|c|c|c|c|c|c|}
\hline 8 & Abertura de Refrigeração & $\mathrm{x}$ & & $x$ & & & & $\mathrm{x}$ & \\
\hline 9 & Luz Dianteira & $\mathrm{x}$ & $\mathrm{x}$ & $\mathrm{x}$ & & $\mathrm{x}$ & & & \\
\hline 10 & Capô & $\mathrm{x}$ & $\mathrm{x}$ & & & & & $\mathrm{x}$ & \\
\hline 11 & Para-brisa & $\mathrm{x}$ & & & & & & & \\
\hline 12 & Teto & $\mathrm{x}$ & & & & $\mathrm{x}$ & & & \\
\hline 13 & Portas & $\mathrm{x}$ & & $\mathrm{x}$ & $\mathrm{x}$ & $\mathrm{x}$ & $\mathrm{x}$ & & $\mathrm{x}$ \\
\hline
\end{tabular}

Fonte: adaptado de Yogasara e Valentino (2017)

O estudo de Sihombing et al. (2017) investigou a relação das palavras kansei "estilo", "conforto", "segurança", "luxuosidade" e "esportividade" com seis modelos de carros lançados no mercado da Malásia entre os anos de 2014 e 2016, e distribuídos nos seguintes segmentos: MPV (Toyota Alphard); sedan (BMW F80, Audi S6); e city (Toyota Etios Liva, Toyota Aygo e Peugeot 108). Concluiu-se que os segmentos MPV e city expressam conforto, enquanto os sedans evocam luxuosidade. E, ainda, as vistas que mais influenciaram a decisão dos 171 participantes foram as laterais e as traseiras. Demograficamente, observou-se que: o gênero exerce maior impacto na vista lateral do segmento MPV; a ocupação influencia a vista lateral da classe city; e a idade tem efeito na vista lateral dos sedans.

Tabela 3 - Segmentos, modelos, vistas e preferência das palavras Kansei

\begin{tabular}{llll}
\hline Segmento & Modelo & Vista & Palavra Kansei \\
\hline MPV & Toyota Alphard & $\begin{array}{l}\text { frontal } \\
\text { lateral } \\
\text { traseira }\end{array}$ & $\begin{array}{l}\text { conforto } \\
\text { conforto } \\
\text { conforto }\end{array}$ \\
\hline Sedan & BMW F80 & frontal & $\begin{array}{l}\text { luxuosidade } \\
\text { estilo } \\
\text { lateral }\end{array}$ \\
& BMW F80 & traseira & luxidade \\
\hline City & Audi S6 & frontal & $\begin{array}{l}\text { conforto } \\
\text { conforto } \\
\text { lateral } \\
\text { traseira }\end{array}$ \\
\hline
\end{tabular}

Fonte: adaptado de Sihombing et al. (2017)

\section{Considerações Finais}

O objetivo deste artigo é contribuir com a investigação da aplicação da metodologia de engenharia Kansei, praticada globalmente, cujo enfoque se dá na identificação da relação qualitativa entre as emoções do usuário e a tradução destas em dados objetivos de parâmetros de projeto e elementos de design automotivo. A revisão da literatura, mesmo que referente apenas ao design de exterior, apresenta um panorama consistente do emprego do método Kansei no contexto 
do design automotivo.

Observa-se que os estudos de caso aqui abordados são apenas divulgados no idioma inglês e, portanto, carecem de uma tradução dos termos empregados neste contexto à língua portuguesa. Com isso, esta revisão visa também fomentar pesquisas nacionais que relacionem a aplicação da metodologia Kansei e o design de exterior automotivo e, consequentemente, colaborar para minimizar a lacuna de investigações brasileiras direcionadas a este fim.

Por meio dos estudos de Fukushima (1995), Ishihara (2005) e Nagamachi (2002), buscou-se também elucidar as oito categorias distintas que compõem o escopo da engenharia Kansei, e são praticadas até hoje, mesmo que para isso tenha sido necessário distanciar-se do escopo automotivo. A partir disso, foi possível correlacionar aqui os sete estudos de caso aos tipos de EK. Importa enfatizar que, na maioria das fontes originais dos referidos estudos de caso, o tipo de metodologia Kansei empregado não é evidenciado pelos autores.

De modo geral, quando se aborda o design automotivo de exterior pela perspectiva das impressões dos potenciais consumidores, observa-se em quatro dos sete artigos investigados Nagamachi (2002); Lai et al. (2005); Shen et al. (2000); Yogasara e Valentino (2017) - um destaque maior para o entendimento de quais partes do exterior do automóvel influenciam a opinião dos usuários, especialmente quanto aos conceitos de "luxo" e "esportividade". Enquanto as pesquisas de Chang et al. (2006) e Kim et al. (2012) enfocam, respectivamente, as questões relacionadas à descrição verbal do carro idealizado e à avaliação por imagens. Já Sihombing et al. (2017) trabalham tanto na relação entre os conceitos "luxo" e "conforto" e as características holísticas das categorias de automóvel, quanto na avaliação por imagens.

Por meio desta revisão, é possível verificar que a complexidade da metodologia KE exige uma cooperação transdisciplinar entre os universos acadêmico e industrial, bem como entre as áreas de humanidades, ciências sociais e ciências naturais, a exemplo dos seguintes estudos: design do exterior frontal e a percepção de consumidores de três países distintos na cooperação entre a empresa norte-americana Sprint PCS junto às universidades Kagawa, Nottingham e ao Instituto Nacional de Tecnologia Kure (Shen, 2000); além da pesquisa de Jindo e Hirasago (1997), em um trabalho conjunto com a Nissan do Japão e a Universidade de Hiroshima, aqui citado mas relacionado ao design automotivo de interior.

Por fim, ressalta-se que o estudo do design de exterior automotivo traz expressivas contribuições à investigação acadêmica, uma vez que a percepção do carro pelo potencial consumidor e as respostas emocionais decorrentes disso são fenômenos de complexa compreensão, e estão significativamente ligados às pesquisas em ergonomia (SHEN et al. 2012). Como aponta o estudo de Shen et al. (2012), o comportamento do usuário é muitas vezes diferente das respostas indicadas em um questionário. Assim, como desdobramento futuro e com o objetivo de mitigar discrepâncias desta natureza, as autoras desta revisão intencionam a adoção de dispositivos não invasivos, direcionados à aferição e à avaliação de parâmetros neurofisiológicos do potencial usuário, em associação ao escopo metodológico da EK. Tal estratégia visa ampliar a compreensão sobre os processos perceptivos, emocionais e cognitivos relacionados à tomada de decisão na escolha de um automóvel, para orientar a melhor definição projetual do time de desenvolvimento de produtos neste âmbito. 


\section{Referências}

BAHN, Sangwoo; LEE, Cheol; NAM, Chang; YUN, Myung. Incorporating Affective Customer Needs for Luxuriousness into Product Design Attributes. Human Factors and Ergonomics in Manufacturing \& Service Industries, v. 19, n.ㅇ 2, p. 105-127, 2009.

CHANG, H-C; LAl, H-H; CHANG, Y-M. Expression modes used by consumers in conveying desire for product form: A case study of a car. International Journal of Industrial Ergonomics, Taiwan, v. 36, p. 3-10, 2006.

FUKUSHIMA, K.; KAWATA, H.; FUJIWARA, Y.; GENNO, H. Human sensory perception oriented image processing in a color copy system. International Journal of Industrial Ergonomics, v. 15, n.․ 1, p. 6374, 1995.

GABARDO, Ana Cláudia. Proposta de aprendizagem da metodologia kansei engineering no curso de design de produto. Dissertação (Mestrado em Design) - Universidade Federal do Paraná, Curitiba, 154f. 2014.

GENTNER, A.; Bouchard, C.; ESQUIVEL, D.; FAVART, C. Mapping a multi sensory identity territory at the early design stage. International Journal of Affective Engineering, v. 12, n. 2 p. 191-200, 2013.

JINDO, Tomio; HIRASAGO, Kiyomi. Application studies to car interior of Kansei engineering. International Journal of Industrial Ergonomics, v. 19, p. 105-114, 1997.

JUNG, G.; KIM, S. M.; KIM, S. Y.; JUNG, E. S.; PARK, S. Effects of Design Factors of the Instrument Cluster Panel on Consumers' Affection. Proceedings of the International MultiConference of Engineers and Computer Scientists IMECS 2010, Hong Kong, v. 3, 2010.

KIM, C.; LEE, C.; LEHTO, M. R.; YUN, M. H. Affective evaluation of user impressions using virtual product prototyping. Human Factors and Ergonomics in Manufacturing \& Service Industries, v. 21 n.. 1, p. 1-13, 2011.

$\mathrm{KIM}$, S.; CHO, Y.; NIKI, K.; YAMANAKA, T. The Relationship between Preference and Kansei Values. Kansei Engineering International Journal, v. 11, n. 4, p. 259-266, 2012.

LAI, H-H.; CHANG, Y-M.; CHANG, H-C. A robust design approach for enhancing the feeling quality of a product: a car profile case study. International Journal of Industrial Ergonomics, v. 35, n. 05 , Taiwan, p. 445-460, 2005.

LOKMAN, A. M. Design \& Emotion: the kansei engineering methodology. Design \& Emotion: the kansei engineering methodology, v. 1, n. 0 1, 2010.

MOHAMED, M. S. S.; MUSTAFA, S. Kansei Engineering Implementation on Car Center Stack Designs. International Journal of Education and Research, v. 2, n. ㅇ 4. p. 355-366, 2014.

NAGAMACHI, Mitsuo. Kansei engineering: A new ergonomic consumer-oriented technology for product development. International Journal of Industrial Ergonomics, v. 15, p. 3-11, 1995.

. Kansei engineering as a powerful consumer oriented technology for product development. Applied Ergonomics, v. 33, n.3, p. 289-294, 2002a.

. Kansei Engineering in Consumer Product Design. Ergonomics in Design: The Quarterly of Human Factors Applications, v. 10, p. 5, 2002b.

. Perspectives and new trend of Kansei/Affective Engineering. 1st European Conference on 
Affective Design and Kansei Engineering \& 10th QMOD Conference, University of Linkoping and Lund University, Helsingborg, 2007.

OKAMOTO, R. H.; NISHINO, T.; NAGAMACHI, M. Comparison between statistical and lower / upper approximations rough sets models for beer can design and prototype evaluation. The 10th International Conference on Quality Management and Operation Development (QMOD'07), Sweden, Linköping University Electronic Press, p. 1650-3740, 2007.

SCHÜTTE, Simon. Designing feelings into products: Integrating kansei engineering methodology in product development. Doctoral Dissertation (Quality and Human System Engineering) - Linköping University, Sweden, 97p. 2002.

SCHÜTTE, S. T. W.; EKLUND, J. AXELSSON, J. R. C.; NAGAMACHI, M. Concepts, methods and tools in kansei engineering. Theoretical Issues in Ergonomics Science, v. 5, n.o 3, p. 214-231, 2004.

SCHÜTTE, S. T. W.; EKLUND, J. Design of rocker switches for work-vehicles-an application of Kansei Engineering. Applied Ergonomics, v. 36, n.ㅇ 5, p. 557-567, 2005.

SHEN, W.; MATSUBARA, Y.; WILSON, J.; NAGAMACHI, M. A Cross-Cultural Study of Vehicle Front Mask Design Using Kansei Engineering Approach. Proceedings of the Human Factors and Ergonomics Society Annual Meeting, v. 44, n.o 33, p. 6-372-6-375, 2000.

SHEN, K. S.; CHEN, K. H.; LIANG, C. C.; PU, W. P.; MA, M. Y. Measuring the Functional and Usable Appeal of Crossover B-Car Interiors. Human Factors and Ergonomics in Manufacturing \& Service Industries, v.00, no 0, p. 1-17, 2012.

SIHOMBING, H.; SYAFIKA, A.; SULAINMAN, S.; SALLEH, M. R.; YAAKOB, M. Y. The product design preferences based on KE: car products in Malasya. Proceedings of Mechanical Engineering Research Day, p. 200-201, 2017.

TANOUE, C.; ISHIZAKA, K.; NAGAMACHI, M. Kansei Egineering: a study on perception of vehicle interior image. International Journal of Industrial Ergonomics, v. 19, p. 115-128, 1997.

VIEIRA, J., Osório, J. M. A., Mouta, S., Delgado, P., Portinha, A., Meireles, J. F., \& Santos, J. A. Kansei engineering as a tool for the design of in-vehicle rubber keypads. Applied ergonomics, v. 61, p. 111, 2017.

YOGASARA, T.; VALENTINO, J. Realizing the Indonesian National car: The design of the $4 \times 2$ wheel drive passenger car exterior using Kansei Eng Type 1. International Journal of Technology, v. 2, p. 338-351, 2017. 